\title{
Biodistribution and Dosimetry Results from a Phase 1 Trial of Therapy with the Antibody-Radionuclide Conjugate ${ }^{177}$ Lu-Lilotomab Satetraxetan
}

\author{
Johan Blakkisrud $^{1}$, Jon Erik Holtedahl ${ }^{1,2}$, Ayca Løndalen ${ }^{3}$, Jostein Dahle ${ }^{4}$, Tore Bach-Gansmo ${ }^{3}$, Harald Holte ${ }^{5}$ \\ Stine Nygaard ${ }^{5}$, Arne Kolstad ${ }^{5}$, and Caroline Stokke ${ }^{1,6}$ \\ ${ }^{I}$ Department of Diagnostic Physics, Division of Radiology and Nuclear Medicine, Oslo University Hospital, Oslo, Norway, and \\ University of Oslo, Oslo, Norway; ${ }^{2}$ Siemens Healthineers, Oslo, Norway; ${ }^{3}$ Division of Radiology and Nuclear Medicine, \\ Oslo University Hospital, Oslo, Norway; ${ }^{4}$ Nordic Nanovector ASA, Oslo, Norway; ${ }^{5}$ Department of Oncology, Radiumhospitalet, \\ Oslo University Hospital, Oslo, Norway; and ${ }^{6}$ Department of Life Sciences and Health, Oslo Metropolitan University, Oslo, Norway
}

\begin{abstract}
${ }^{177}$ Lu-lilotomab satetraxetan is a novel antibody-radionuclide conjugate currently in a phase $1 / 2$ a first-in-humans dose escalation trial for patients with relapsed CD37-positive indolent non-Hodgkin lymphoma. The aim of this study was to investigate biodistribution and absorbed doses to organs at risk. Methods: In total, 7 patients treated with ${ }^{177} \mathrm{Lu}$-lilotomab satetraxetan were included for dosimetry. Patients were grouped on the basis of 2 different predosing regimens (with and without predosing with $40 \mathrm{mg}$ of lilotomab) and were treated with different levels of activity per body weight $(10,15$, and 20 $\mathrm{MBq} / \mathrm{kg}$ ). All patients were pretreated with rituximab. Serial planar and SPECT/CT images were used to determine time-activity curves and patient-specific masses for organs with ${ }^{177} \mathrm{Lu}$-lilotomab satetraxetan uptake. Doses were calculated with OLINDA/EXM. Results: The organs (other than red bone marrow and tumors) with distinct uptake of ${ }^{177}$ Lu-lilotomab satetraxetan were the liver, spleen, and kidneys. The highest uptake was found in the spleen, with doses ranging from 1.54 to $3.60 \mathrm{mGy} / \mathrm{MBq}$. The liver received $0.70-1.15 \mathrm{mGy} / \mathrm{MBq}$. The kidneys received the lowest dose of the source organs investigated, $0.16-0.79 \mathrm{mGy} / \mathrm{MBq}$. No statistically significant differences in softtissue absorbed doses were found between the two predosing regimens. The whole-body dose ranged from 0.08 to $0.17 \mathrm{mGy} / \mathrm{MBq}$. Conclusion: The biodistribution study for patients treated with ${ }^{177} \mathrm{Lu}-$ lilotomab satetraxetan revealed the highest physiologic uptake to be in the liver and spleen (besides the red marrow). For all treatment levels investigated, the absorbed doses were found to be modest when compared with commonly assumed tolerance limits.
\end{abstract}

Key Words: biodistribution; antibody-radionuclide conjugate; nonHodgkin lymphoma; internal dosimetry

J Nucl Med 2018; 59:704-710

DOI: 10.2967/jnumed.117.195347

\section{$\mathbf{M}$}

onoclonal antibodies have been used for many years in the targeting of cancer cells. Arming antibodies with radioisotopes is a means to direct ionizing radiation selectively at tumor sites in

Received May 5, 2017; revision accepted Aug. 1, 2017.

For correspondence or reprints contact: Johan Blakkisrud, Department of Diagnostic Physics, Oslo University Hospital, Gaustad sykehus bygg 20, P.O. Box 4959 Nydalen, 0424 Oslo, Norway.

E-mail: johbla@ous-hf.no

Published online Aug. 28, 2017.

COPYRIGHT (C 2018 by the Society of Nuclear Medicine and Molecular Imaging. order to deliver clinically effective amounts of radiation to tumors while minimizing dose to normal tissues. The treatments are suited for systemic diseases, especially for the radiosensitive lymphomas $(1,2)$. Two radioimmunoconjugates have been approved for treatment of non-Hodgkin lymphoma, ${ }^{131}$ I-tositumomab (Bexxar; GlaxoSmithKline LLC) and ${ }^{90}$ Y-ibritumomab tiuxetan (Zevalin; Spectrum Pharmaceuticals), both based on the CD20 antibody (3). Considering that these 2 radioimmunoconjugates are most often used to treat patients who have already received rituximab, which also targets CD20, it is of interest to explore a new conjugate that targets a different antigen. The CD37 antigen is abundantly expressed on B cells but is absent from plasma cells and normal stem cells $(4,5)$. Therefore, CD37 seems to be an appropriate therapeutic target in patients with relapsed B-cell-derived malignancies.

Radioimmunotherapy with CD37 as a target has previously been explored clinically using a ${ }^{131} \mathrm{I}$-labeled murine monoclonal antibody (MB-1) (6,7). A higher degree of internalization and degradation of ${ }^{131}$ I-labeled radioimmunoconjugate was found for CD37 than for CD20 (8). The potency of radioimmunotherapy against the internalizing antigen CD37 might have been underestimated by the use of a nonresidualizing radionuclide in the early studies with ${ }^{131} \mathrm{I}-\mathrm{MB}-1$ (9).

In recent years, ${ }^{177} \mathrm{Lu}$ has emerged as a favorable isotope for molecular radiotherapy. It has been used in clinical trials for diseases including adult neuroendocrine disease, prostate cancer, and colorectal carcinoma $(10,11)$ and is routinely used in peptide receptor radionuclide therapy (12). The $\beta$-particles emitted by ${ }^{177} \mathrm{Lu}$ have a mean energy of $0.13 \mathrm{MeV}$ (half-life, $6.7 \mathrm{~d}$ ), and ${ }^{177} \mathrm{Lu}$ emits mainly $113-\mathrm{keV}(6 \%)$ and $208-\mathrm{keV}(11 \%)$ photons (13). This is a major advantage, since these photons contribute little to excess radiation but make posttherapy imaging feasible. Absorbed doses can therefore be retrospectively measured by quantification of the images. ${ }^{177} \mathrm{Lu}$-lilotomab satetraxetan (Betalutin; Nordic Nanovector ASA) is a novel antibody-radionuclide conjugate that targets CD37 and is currently under investigation in the phase 1/2a LYMRIT-37-01 trial for patients with relapsed CD37-positive B-cell non-Hodgkin lymphoma (14). Red marrow and tumor doses have been previously reported for ${ }^{177} \mathrm{Lu}$-lilotomab satetraxetan $(15,16)$.

Here, 7 of the LYMRIT-37-01 patients treated with ${ }^{177} \mathrm{Lu}$-lilotomab satetraxetan were included in a biodistribution and dosimetry protocol and subjected to more extensive imaging over the first week after administration. The aim of this work was to determine normaltissue absorbed doses for ${ }^{177} \mathrm{Lu}$-lilotomab satetraxetan using the imaging data from this first-in-humans trial. 


\section{MATERIALS AND METHODS}

Patient Inclusion and Treatment

The phase 1 LYMRIT-37-01 trial is a nonrandomized singleinjection dose-finding study for treatment of relapsed indolent CD37positive non-Hodgkin lymphoma. The trial was approved by the regional ethical committee, and the patients gave written informed consent. They received a fixed amount of radioactivity per unit body weight (Table 1). Approximately $4-10 \mathrm{mg}$ of ${ }^{177} \mathrm{Lu}$-lilotomab satetraxetan was injected in a typical patient (75-kg body mass, $15 \mathrm{MBq} / \mathrm{kg}$ ), resulting in a specific activity of approximately 120-300 MBq per milligram of antibody. Patients in arm 1 (patients 2, 3, and 5) were predosed with $40 \mathrm{mg}$ of lilotomab (cold CD37 antibody); the patients in arm 2 (patients 13,14, and 15), as well as patient 1 , did not receive this predosing (Fig. 1).

\section{Image Acquisition}

The patients underwent planar whole-body (WB) imaging and SPECT/CT at multiple time points after administration of ${ }^{177} \mathrm{Lu}-\mathrm{lilo}-$ tomab satetraxetan (Fig. 1). Scans were obtained on a dual-head Siemens Symbia T16 SPECT/CT device equipped with a medium-energy collimator using $2 \times 32$ projections, each of 45-s frame length in a noncircular orbit. A $20 \%$ window around $208 \mathrm{keV}$, along with a low-scatter window of $20 \%$ of the $208-\mathrm{keV}$ peak just below the main energy window, was applied. The WB images were acquired with a $256 \times 1,024$ matrix and a scan speed of $5 \mathrm{~cm} / \mathrm{min}$. A thin vacuum mattress on the examination table was used as a means of fixation, to ensure that the patients were in the same position each time. The low-dose CT scan was acquired once over the total body length, such that the scan could be used for attenuation correction of the WB images. As a constancy control, a vial with a known activity of ${ }^{177} \mathrm{Lu}$ was placed alongside the patients.

For patients 13 and 14 , urine was collected separately at each micturition during the first $2 \mathrm{~h}$, and then all urine micturated over the next $18-22 \mathrm{~h}$ was collected together.

\section{Phantom Measurements and Scanner Calibration}

The scanner was calibrated using a large water-filled phantom with a smaller insert containing ${ }^{177} \mathrm{Lu}$-lilotomab satetraxetan $(103.6 \mathrm{MBq}$ in $1.2 \mathrm{~L}$ ). A calibration factor of $39.1 \mathrm{~Bq} /$ count on the SPECT images was found from a volume-of-interest well inside the insert. This factor was validated by quantifying the known activities in an anthropomorphic torso phantom (model ECT/TOR/P; Data Spectrum Corp.). The phantom contained 3 inserts-tumor (20.5 MBq in $5 \mathrm{~mL}$ ), kidney

TABLE 1

Patients Included in Biodistribution and Dosimetry Study

\begin{tabular}{cccccc}
\hline $\begin{array}{c}\text { Patient } \\
\text { no. }\end{array}$ & Sex & $\begin{array}{c}\text { Pretreatment } \\
\text { and } \\
\text { predosing }\end{array}$ & $\begin{array}{c}\text { Treatment } \\
\text { level } \\
(\mathrm{MBq} / \mathrm{kg})\end{array}$ & $\begin{array}{c}\text { Body } \\
\text { weight } \\
\text { (kg) }\end{array}$ & $\begin{array}{c}\text { Injected } \\
\text { activity } \\
(\mathrm{MBq})\end{array}$ \\
\hline 1 & $\mathrm{~F}$ & $\mathrm{R}$ & 10 & 118 & 1,102 \\
\hline 2 & $\mathrm{M}$ & $\mathrm{R}+\mathrm{L}$ & 10 & 103 & 1,036 \\
3 & $\mathrm{M}$ & $\mathrm{R}+\mathrm{L}$ & 10 & 73 & 746 \\
\hline 5 & $\mathrm{M}$ & $\mathrm{R}+\mathrm{L}$ & 20 & 97 & 1,982 \\
13 & $\mathrm{M}$ & $\mathrm{R}$ & 15 & 94 & 1,416 \\
14 & $\mathrm{~F}$ & $\mathrm{R}$ & 15 & 71 & 1,013 \\
15 & $\mathrm{M}$ & $\mathrm{R}$ & 10 & 113 & 1,137 \\
\hline
\end{tabular}

$\mathrm{R}=$ pretreated with rituximab; $\mathrm{R}+\mathrm{L}=$ pretreated with rituximab and predosed with lilotomab.

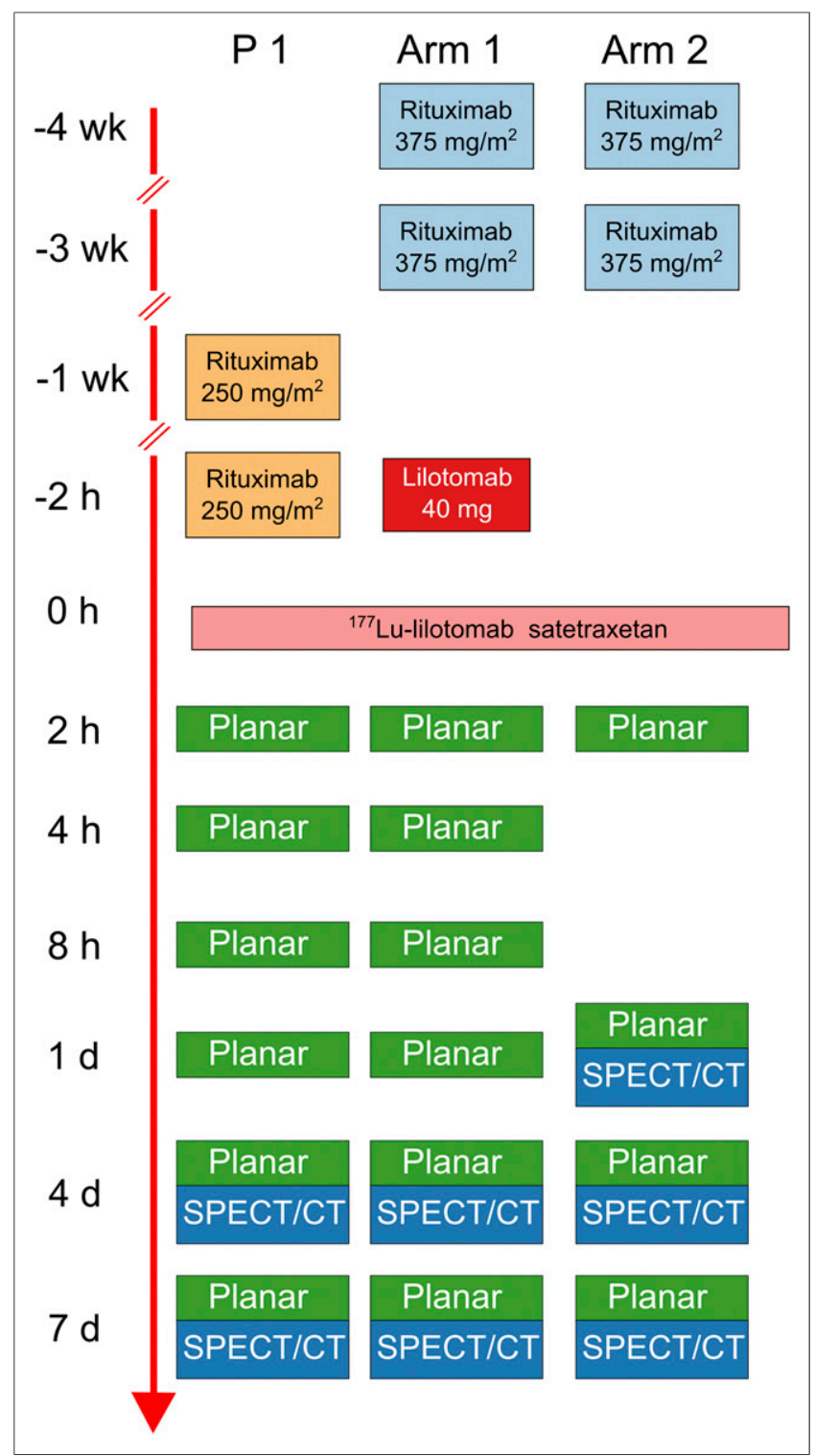

FIGURE 1. Pretreatment, predosing, and imaging protocol. Different predosing and pretreatment regimens are shown in parallel. Zerohour time point of nonlinear time line is set according to injection of ${ }^{177}$ Lu-lilotomab satetraxetan. P $1=$ patient 1 ; planar $=$ WB planar scintigraphy.

(13.6 MBq in $113 \mathrm{~mL}$ ), and spleen (50.0 MBq in $106 \mathrm{~mL}$ )—each filled with ${ }^{177} \mathrm{Lu}$-lilotomab satetraxetan. The measurements resulted in relative errors of $3.3 \%,-2.1 \%$, and $0.4 \%$, respectively.

\section{Data Analysis and Quantification}

For SPECT/CT images, the activities in the organs with distinct uptake (source organs) were found by manually drawing volumes of interest using PMOD, version 3.6 (PMOD Technologies Ltd.). SPECT-derived values were used primarily to determine organ kinetics. For time points at which only planar imaging had been performed, the planar imaging-derived organ counts were adjusted according to the ratio between the planar counts and the SPECT activity values on day 4 . The time-activity curves were calculated by interpolation between time points with a trapezoidal fit, followed by extrapolation after the last time point to a monoexponential curve 
based on days 4 and 7. Activity at the first imaging point was assumed to represent the initial activity in a region. The time-integrated activity coefficients were calculated from the area under the time-activity curves. Time-integrated activity coefficients for red marrow were retrieved from a separate work (15). Simulations investigating fewer imaging time points were performed by integrating time-activity curves after removal of time-activity points. The individual organ volumes were found from delineating the organs using $\mathrm{CT}$ images from day 4 . A mass density of $1 \mathrm{~g} / \mathrm{mL}$ was assumed.

For planar images, an in-house-made MATLAB program (version 2015a, The MathWorks, Inc.) was applied to automatically coregister the images using an intensity-based registration. Counts in the organs at different time points were found by manually drawing a region of interest around them on geometric mean images. This was done by 3 independent analysts, and the mean of their results was calculated. To correct for background activity, a region near the organ region of interest was used for subtraction of counts. For the kidneys, the background-toorgan activity ratios were generally low, occasionally resulting in negative counts, and therefore background correction for the kidneys was omitted. Attenuation-corrected planar images were also made and used for a parallel organ dose calculation.

The WB activity at $2 \mathrm{~h}$ was assumed to represent the total injected activity. WB activities at later time points were calculated using the planar images and normalization with the first time point. Timeactivity curve fitting was performed monoexponentially.

\section{Dosimetry}

Absorbed doses were calculated using the MIRD scheme (17). The individual time-integrated activity coefficients for each source organ (including WB and red marrow) were imported to OLINDA/EXM (version 1.1, Vanderbilt University) for dose calculations. Standard adult male and female phantoms were used. The individual masses of the source organs were used as input. WB mass was set to patientspecific values without scaling the mass of the remaining organs. For the rest of the organs, the default phantom values in OLINDA/EXM were used.

\section{Statistics}

Absorbed doses for patients with and without predosing were compared using a 2-sided Student $t$ test.

\section{RESULTS}

\section{Biodistribution and Absorbed Doses}

Tumor, spleen, liver, bone marrow, and kidneys were determined visually to have distinct uptake of ${ }^{177} \mathrm{Lu}$-lilotomab satetraxetan and were categorized as source organs (Fig. 2). The effective WB half-life of ${ }^{177} \mathrm{Lu}$-lilotomab satetraxetan was similar across the population (time-activity curves in Fig. 3D), with a mean of $82 \mathrm{~h}$ (ranging from 72 to $95 \mathrm{~h}$ ). The activity excreted via urine before the first planar scan was 21 and $9 \mathrm{MBq}(1.5 \%$ and $0.9 \%$ of the injected activity) for patients 13 and 14 , respectively. These data indicate that only marginal errors are introduced by assuming that all injected activity is retained at the first WB imaging time point, and this assumption was therefore used for all patients.

The spleen received the highest organ doses, with a mean of $3.57 \mathrm{~Gy}$, and the maximum observed value was $6.50 \mathrm{~Gy}$ (Table 2). The liver and kidneys received a maximum dose of 2.00 and 0.62 Gy, respectively. No adverse events related to kidney or liver function have been reported during a follow-up of 15-50 mo for patients who received ${ }^{177} \mathrm{Lu}$-lilotomab satetraxetan.

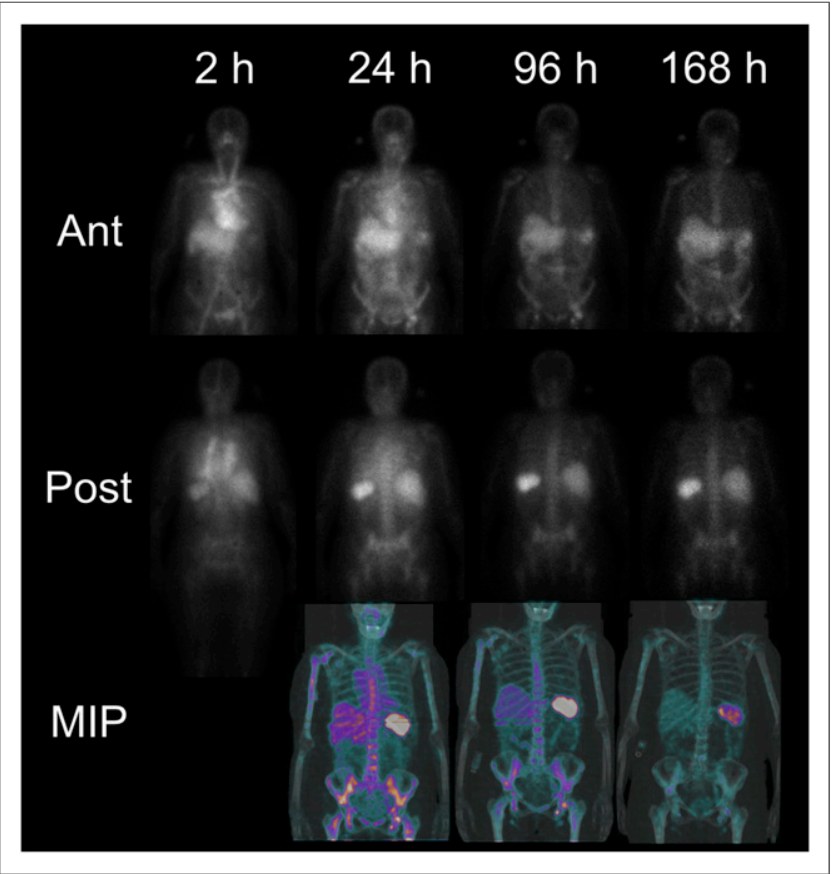

FIGURE 2. Planar (anterior [Ant] and posterior [Post], 4 time points) and SPECT/CT images (maximum-intensity projection [MIP], 3 time points) of patient 14 after injection of ${ }^{177} \mathrm{Lu}$-lilotomab satetraxetan. Activity in blood can be seen at early time points, with gradual uptake followed by washout in liver, spleen, and bone marrow. Uptake in inguinal tumors is also visible.

Table 3 sums the absorbed doses per unit administered activity for the 2 arms, here including patient 1 with arm 2 . The average values across all patients were determined to be 2.92, 0.92, and $0.35 \mathrm{mGy} / \mathrm{MBq}$ for the spleen, liver, and kidneys, respectively. WB doses were 0.14 and $0.12 \mathrm{mGy} / \mathrm{MBq}$ for predosed patients and nonpredosed patients, respectively. A conservative calculation using reference WB masses instead of patient-specific values would change the WB doses to 0.16 and $0.15 \mathrm{mGy} / \mathrm{MBq}$, respectively. The difference in dose per injected activity for the spleen, kidneys, liver, and WB between predosed and nonpredosed patients was not significant (lowest $P=0.24$ ). Predosed patients received a lower dose to the red marrow than nonpredosed patients $(P=0.05)$. For the other organs, which had less uptake of ${ }^{177} \mathrm{Lu}-$ lilotomab satetraxetan, the doses were calculated using OLINDA/ EXM. The doses to these organs are primarily the result of $\beta$-radiation from the assumed uniform remaining WB distribution of activity not assigned to the source organs.

\section{Methodologic Evaluations}

Various aspects of the dose calculation were investigated. Care was taken to apply the same resulting methods to all patients.

The common assumption of a constant ratio between the SPECT and planar images over time was explored using the additional SPECT data from day 7. An average agreement of $9 \%$ $( \pm 5 \%$ ) between the planar value on day 7 (corrected by the ratio of planar-to-SPECT on day 4) and the direct measurement from SPECT on day 7 was found in patients from arms 1 and 2. This deviation was considered to contribute to substantial errors in the time-activity curve calculation; therefore, SPECT-derived data were primarily used for all patients. 


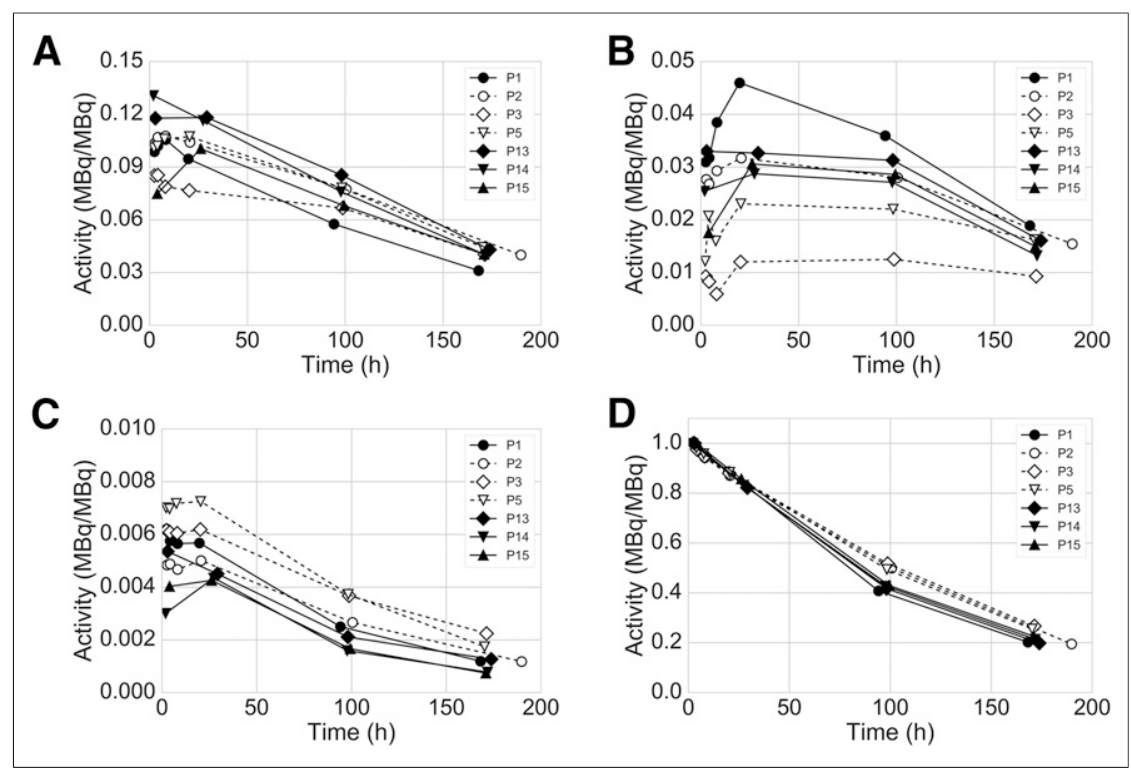

FIGURE 3. Time-activity curves showing uptake and clearance in liver, spleen, and kidney (A, $B$, and $C$, respectively) and WB (D) for individual patients. Measured activity was normalized by injected activity. Decay correction was not performed.

Time-integrated activity coefficients for the source organs in arm 1 were compared using attenuation-corrected versus nonattenuation-corrected planar images; the deviations were within $1 \%$. For WB, the deviations were within $3 \%$. Because the relative planar values are adjusted by absolute organ values obtained from SPECT/CT (or injected activity for WB) and the planar correction matrix is constant over time, the minor differences between corrected and noncorrected image sets can most likely be explained by alignment errors in the attenuation correction matrix. Attenuation correction of planar images was therefore omitted for all patients.

Supplemental Figure 1 (supplemental materials are available at http://jnm.snmjournals.org) shows what the difference in the calculated doses for arm 1 patients would be if a reduced number of imaging time points had been obtained. Removal of the 4- and 8-h time points led to deviations no greater than $1 \%$, and these 2 imaging points were therefore omitted in arm 2.

\section{DISCUSSION}

This biodistribution and dosimetry study was a part of a phase 1/2a trial of ${ }^{177} \mathrm{Lu}$-lilotomab satetraxetan for treatment of nonHodgkin lymphoma, which is the first-in-humans trial of this antibody-radionuclide conjugate. For all treatment levels and the 2 predosing regimens investigated, the absorbed doses to the liver, spleen, and kidneys were within commonly assumed tolerance levels. Accordingly, no signs of nonhematologic toxicities were observed after treatment.

${ }^{177} \mathrm{Lu}$-lilotomab satetraxetan was seen in the blood and heart at $2 \mathrm{~h}$ after injection, and uptake in the liver and kidneys was also visible at this early time point (Fig. 2). Peak spleen levels can be estimated to occur at a later time point some days after administration of ${ }^{177} \mathrm{Lu}$-lilotomab satetraxetan (Fig. 3). A relatively low proportion of the activity distributed to the kidneys, compared with some previously investigated radioimmunoconjugates, probably demonstrating the larger molecular weight of the antibody- radionuclide conjugate or stronger binding of the radiometal ${ }^{177} \mathrm{Lu}$ to the antibody.

Preclinical studies suggested the red marrow, liver, spleen, and kidneys to be the organs receiving the higher doses, and red marrow to be the critical organ, for ${ }^{177} \mathrm{Lu}$-lilotomab satetraxetan treatment (14). The clinical dosimetry results fit well with the preclinical findings. For the liver and kidneys, the absorbed doses were far below the external-beam radiation therapy tolerance levels and the suggested levels from ${ }^{177} \mathrm{Lu}$-DOTATATE treatment $(18,19)$. However, the variances in dose rate, energy, and other factors for different treatments suggest that tolerance limits should be verified empirically for new therapies. Preliminary clinical findings for ${ }^{177} \mathrm{Lu}$-lilotomab satetraxetan have shown that adverse events are limited to hematologic toxicities (20). The observed correlation between red marrow dose and thrombocytopenia for these patients was previously discussed (15). The lack of other adverse events supports the finding that the reported kidney and liver doses are well within tolerance limits for ${ }^{177} \mathrm{Lu}$-lilotomab satetraxetan therapy. Radiation-induced effects of spleen irradiation may include hematologic effects, and investigations for ${ }^{177} \mathrm{Lu}$-DOTATATE treatment have suggested that spleen doses can contribute to hematologic toxicity (21). However, the evidence indicates that doses no higher than 6.5 Gy (maximum dose found in the present study; Table 2) are too low to introduce such effects $(21,22)$. Isolating theoretic contributions from spleen irradiation to the hematologic adverse events is therefore difficult for ${ }^{177} \mathrm{Lu}$-lilotomab satetraxetan treatment.

The organs receiving the highest doses in radioimmunoconjugate therapy for lymphoma are typically the spleen, liver, kidneys, and bone marrow (6,23-26). Two anti-CD20 radioimmunoconjugates, ${ }^{90}$ Y-ibritumomab tiuxetan and ${ }^{131}$ I-tositumomab, have been approved by the Food and Drug Administration for treatment of non-Hodgkin lymphoma. It is therefore relevant to compare doses between these treatments and ${ }^{177} \mathrm{Lu}$-lilotomab satetraxetan therapy. ${ }^{131}$ I-tositumomab is administered to limit the WB dose to 75 cGy (27). The amount of ${ }^{90}$ Y-ibritumomab tiuxetan is administrated on the basis of patient body weight $(15 \mathrm{MBq} / \mathrm{kg})$, limited by a maximum activity of $1,200 \mathrm{MBq}(28)$. The expected organ and WB doses for a ${ }^{90}$ Y-ibritumomab tiuxetan, ${ }^{131}$ I-tositumomab, and ${ }^{177} \mathrm{Lu}$-lilotomab satetraxetan treatment regimen for a hypothetical $80-\mathrm{kg}$ patient are compared in Table 4 . The ${ }^{177} \mathrm{Lu}$-lilotomab satetraxetan dosage level is based on preliminary clinical results and maximum tolerated activity levels. The comparison indicates that ${ }^{177} \mathrm{Lu}$-lilotomab satetraxetan doses will mostly be equal to or lower than typical ${ }^{90}$ Y-ibritumomab tiuxetan or ${ }^{131}$ I-tositumomab absorbed doses. However, a more clinically relevant comparison could be considered to compare absorbed doses between isoeffective treatment administrations, instead of simply the prescribed values, and the results should therefore be interpreted with care.

For targeted molecular radiotherapies of non-Hodgkin lymphoma, patient-specific dose regimens based on administration of a pretherapy tracer activity have been explored. The therapeutic amounts of ${ }^{131}$ I-tositumomab to be delivered were calculated from 
TABLE 2

Individual Absorbed Dose, Mass, and Time-Integrated Activity Coefficient for Target Organs

\begin{tabular}{|c|c|c|c|c|}
\hline Organ & Patient no. & Mass (g) & TIAC (h) & Dose (Gy) \\
\hline \multirow[t]{7}{*}{ Liver } & 001 & 1,876 & 14.62 & 0.77 \\
\hline & 002 & 2,405 & 19.84 & 0.77 \\
\hline & 003 & 1,295 & 16.74 & 0.86 \\
\hline & 005 & 1,705 & 19.38 & 2.00 \\
\hline & 013 & 1,690 & 20.03 & 1.49 \\
\hline & 014 & 1,687 & 19.05 & 1.04 \\
\hline & 015 & 2,030 & 17.8 & 0.90 \\
\hline \multirow[t]{7}{*}{ Spleen } & 001 & 262 & 7.97 & 2.92 \\
\hline & 002 & 406 & 7.15 & 1.60 \\
\hline & 003 & 100 & 4.15 & 2.69 \\
\hline & 005 & 194 & 7.34 & 6.50 \\
\hline & 013 & 170 & 6.76 & 4.89 \\
\hline & 014 & 152 & 5.55 & 3.21 \\
\hline & 015 & 187 & 5.96 & 3.15 \\
\hline \multirow[t]{7}{*}{ Kidney } & 001 & 204 & 0.67 & 0.33 \\
\hline & 002 & 233 & 0.70 & 0.29 \\
\hline & 003 & 117 & 1.05 & 0.59 \\
\hline & 005 & 270 & 0.93 & 0.62 \\
\hline & 013 & 211 & 0.69 & 0.42 \\
\hline & 014 & 147 & 0.47 & 0.30 \\
\hline & 015 & 282 & 0.47 & 0.18 \\
\hline
\end{tabular}

TIAC = time-integrated activity coefficient.

Different shadings separate pretreatment and predosing regimens.

the tracer biodistribution for each patient. However, for ${ }^{90} \mathrm{Y}-$ ibritumomab tiuxetan, a surrogate ${ }^{111}$ In-ibritumomab tiuxetan tracer did not estimate absorbed radiation doses that were correlated with the toxicity of the treatment. Doses based on body weight and platelet counts were therefore preferred (29). The low $\gamma$-yield of ${ }^{177} \mathrm{Lu}$ is sufficient for posttherapy imaging but discourages the use of a tracer amount of ${ }^{177} \mathrm{Lu}$-lilotomab satetraxetan before a treatment to calculate the maximum tolerated activity. A possible solution could be to substitute a more $\gamma$-intense isotope or a positron emitter for the ${ }^{177} \mathrm{Lu}$.

In the present study, quantification was predominantly based on SPECT/CT, as the quantifications of phantom insertions solely on planar images demonstrated deviations of up to $35 \%$ (data not shown: background- and attenuation-corrected geometric mean images with use of a calibration source). Differences between a 2- and 3-dimensional protocol for ${ }^{90}$ Y-ibritumomab tiuxetan have been investigated, and systematically lower 3-dimensionally derived doses were found for the liver and spleen (30). The authors argued that although a supporting single SPECT/CT scan improved the accuracy of the calculation, a fully 3-dimensional approach should be preferred. This is also our conclusion for ${ }^{177} \mathrm{Lu}$-lilotomab satetraxetan, given the observed inconsistent ratio of planar imaging-derived activity to SPECT-derived activity. The latest MIRD pamphlet also recommends SPECT/CT for dosimetry of ${ }^{177} \mathrm{Lu}$-based therapies (31).
The doses to the liver, spleen, kidney, and WB did not significantly differ between the predosed (40 $\mathrm{mg}$ of lilotomab) and the nonpredosed groups. Mean kidney doses were lower in nonpredosed patients, and spleen doses tended to be higher. This difference could be explained by higher uptake of ${ }^{177} \mathrm{Lu}$-lilotomab satetraxetan in some organs (including the spleen and red marrow), leading to lower cumulated blood activities, and lower renal excretion in the nonpredosed group. A significant difference in red marrow absorbed dose between predosed and nonpredosed patients was observed both for the current group of patients and for a larger group discussed in our previous work (15). Predosing as a means to improve biodistribution has been used for other antibody-radionuclide conjugates. The amount of predosing investigated in the current study ( $40 \mathrm{mg}$ of lilotomab) is modest compared with the amounts used for ${ }^{90} \mathrm{Y}$-ibritumomab tiuxetan ( $250 \mathrm{mg}$ of ibritumomab/m ${ }^{2}$ ) and ${ }^{131}$ I-tositumomab $(450 \mathrm{mg}$ of tositumomab) $(28,32)$. However, on the basis of in vitro results

TABLE 3

Absorbed Doses to All Organs for Different Treatment Regimens

\begin{tabular}{lcc}
\hline \multicolumn{1}{c}{ Organ } & $\begin{array}{c}\text { With lilotomab } \\
\text { predosing }\end{array}$ & $\begin{array}{c}\text { Without lilotomab } \\
\text { predosing }\end{array}$ \\
\hline Adrenals & $0.12(0.10-0.14)$ & $0.09(0.07-0.12)$ \\
\hline Brain & $0.10(0.08-0.13)$ & $0.07(0.05-0.10)$ \\
\hline Breasts & $0.10(0.08-0.12)$ & $0.07(0.05-0.10)$ \\
\hline Gallbladder wall & $0.12(0.10-0.14)$ & $0.09(0.07-0.12)$ \\
\hline Lower large & $0.11(0.09-0.13)$ & $0.08(0.06-0.11)$ \\
\hline intestine wall & & \\
\hline Small intestine & $0.11(0.09-0.13)$ & $0.08(0.06-0.11)$ \\
\hline Stomach wall & $0.11(0.09-0.13)$ & $0.08(0.06-0.11)$ \\
\hline Upper large & $0.11(0.09-0.14)$ & $0.08(0.06-0.11)$ \\
\hline intestine wall & & \\
\hline Heart wall & $0.11(0.09-0.13)$ & $0.08(0.06-0.11)$ \\
\hline Kidneys & $0.46(0.28-0.79)$ & $0.27(0.16-0.30)$ \\
\hline Liver & $0.97(0.74-1.15)$ & $0.89(0.70-1.05)$ \\
\hline Lungs & $0.11(0.09-0.13)$ & $0.08(0.06-0.11)$ \\
\hline Muscle & $0.10(0.08-0.13)$ & $0.08(0.06-0.10)$ \\
\hline Ovaries & $0.11(0.09-0.13)$ & $0.08(0.06-0.11)$ \\
\hline Pancreas & $0.12(0.10-0.14)$ & $0.1(0.07-0.12)$ \\
\hline Osteogenic cells & $0.50(0.31-0.70)$ & $0.81(0.63-1.03)$ \\
\hline Red marrow & $0.91(0.63-1.18)$ & $1.51(1.39-1.78)$ \\
\hline Skin & $0.10(0.08-0.12)$ & $0.07(0.05-0.09)$ \\
\hline Spleen & $2.81(1.54-3.60)$ & $3.01(2.65-3.45)$ \\
\hline Testes & $0.10(0.08-0.13)$ & $0.07(0.05-0.10)$ \\
\hline Thymus & $0.11(0.09-0.13)$ & $0.08(0.06-0.10)$ \\
\hline Thyroid & $0.10(0.08-0.13)$ & $0.07(0.05-0.10)$ \\
\hline Urinary & $0.11(0.09-0.13)$ & $0.08(0.06-0.10)$ \\
\hline WB & $0.11(0.09-0.13)$ & $0.08(0.06-0.11)$ \\
\hline
\end{tabular}

Data are mean $\mathrm{mGy} / \mathrm{MBq}$, followed by range in parentheses. 
TABLE 4

Doses for ${ }^{90}$ Y-Ibritumomab Tiuxetan and ${ }^{131}$ I-Tositumomab Compared with ${ }^{177}$ Lu-Lilotomab Satetraxetan

\begin{tabular}{|c|c|c|c|}
\hline Organ & ${ }^{90}$ Y-ibritumomab tiuxetan (Gy)* & ${ }^{131}$ I-tositumomab (Gy) ${ }^{\dagger}$ & $\begin{array}{c}{ }^{177} \text { Lu-lilotomab satetraxetan } \\
(20 \mathrm{MBq} / \mathrm{kg})+\text { lilotomab } \\
\text { predosing (Gy) }\end{array}$ \\
\hline Liver & 5.76 & 2.56 & 1.55 \\
\hline Spleen & 11.28 & 3.56 & 4.50 \\
\hline Kidney & 0.12 & 6.10 & 0.74 \\
\hline WB & 0.60 & 0.75 & 0.22 \\
\hline $\begin{array}{l}\text { Red marrow or } \\
\text { marrow space }\end{array}$ & 1.56 & 2.03 & 1.46 \\
\hline
\end{tabular}

${ }^{*}$ An $80-\mathrm{kg}$ patient and a $15 \mathrm{MBq} / \mathrm{kg}$ administered activity are assumed (28).

${ }^{\dagger}$ An administered activity to limit WB dose to 0.75 Gy is assumed (32).

(data not shown), the current predosing with lilotomab is 2-10 times the required amount to block $95 \%-97 \%$ of CD37-positive cells. In this clinical trial, predosing with $40 \mathrm{mg}$ of lilotomab (410 times the amount of administered ${ }^{177} \mathrm{Lu}$-lilotomab satetraxetan) significantly lowered red marrow doses. The optimal amount of predosing for protection of red marrow is currently under investigation. Although it would be interesting to further investigate potential differences for the liver, spleen, and kidneys using a larger number of patients, the clinical need is lessened by the observation that these organs (unlike red marrow) are unlikely to be critical organs for the ${ }^{177} \mathrm{Lu}$-lilotomab satetraxetan treatment.

\section{CONCLUSION}

Normal-tissue doses for the liver, spleen, kidney, and WB were lower than the assumed tolerance limits for patients treated with up to the maximum tolerated activities of ${ }^{177} \mathrm{Lu}$-lilotomab satetraxetan as defined by myelosuppression. Methodologic investigations suggest a fully 3-dimensional imaging-based approach to be the most accurate. There were no significant differences in absorbed dose to soft-tissue organs between patients with and without predosing with $40 \mathrm{mg}$ of lilotomab. Our findings support that red marrow is the critical organ for ${ }^{177} \mathrm{Lu}$-lilotomab satetraxetan therapy and that monitoring the dose to soft-tissue organs may be redundant in ordinary clinical practice.

\section{DISCLOSURE}

The LYMRIT-37-01 study is sponsored by Nordic Nanovector ASA. Johan Blakkisrud is supported by grants from the SouthEastern Norway Regional Health Authority. Harald Holte and Arne Kolstad were both supported in part by grants from the Norwegian Cancer Society. Arne Kolstad is a member of the Scientific Advisory Board of Nordic Nanovector. Jostein Dahle is an employee and shareholder of Nordic Nanovector ASA. No other potential conflict of interest relevant to this article was reported.

\section{ACKNOWLEDGMENTS}

Arne Skretting drafted the initial biodistribution and dosimetry protocol, and we greatly acknowledge his work. We thank the personnel at the nuclear medicine section of Oslo University Hospital for technical assistance with the acquisitions.

\section{REFERENCES}

1. Dewaraja YK, Schipper MJ, Shen J, et al. Tumor-absorbed dose predicts progression-free survival following ${ }^{131} \mathrm{I}$-tositumomab radioimmunotherapy. $\mathrm{J} \mathrm{Nucl}$ Med. 2014;55:1047-1053.

2. Lowry L, Smith P, Qian W, et al. Reduced dose radiotherapy for local control in non-Hodgkin lymphoma: a randomised phase III trial. Radiother Oncol. 2011;100: 86-92.

3. Jacene HA, Filice R, Kasecamp W, Wahl RL. Comparison of ${ }^{90}$ Y-ibritumomab tiuxetan and ${ }^{131}$ I-tositumomab in clinical practice. J Nucl Med. 2007;48:17671776 .

4. Dahle J, Repetto-Llamazares AHV, Mollatt CS, et al. Evaluating antigen targeting and anti-tumor activity of a new anti-CD37 radioimmunoconjugate against non-Hodgkin's lymphoma. Anticancer Res. 2013;33:85-95.

5. Link MP, Bindl J, Meeker TC, et al. A unique antigen on mature B cells defined by a monoclonal antibody. J Immunol. 1986;137:3013-3018.

6. Kaminski MS, Fig LM, Zasadny KR, et al. Imaging, dosimetry, and radioimmunotherapy with iodine 131-labeled anti-CD37 antibody in B-cell lymphoma. J Clin Oncol. 1992;10:1696-1711.

7. Press OW, Eary JF, Badger CC, et al. Treatment of refractory non-Hodgkin's lymphoma with radiolabeled MB-1 (anti-CD37) antibody. J Clin Oncol. 1989; 7:1027-1038.

8. Press OW, Eary JF, Appelbaum FR, et al. Radiolabeled-antibody therapy of B-cell lymphoma with autologous bone marrow support. N Engl J Med. 1993;329: 1219-1224.

9. Repetto-Llamazares A, Abbas N, Bruland ØS, Dahle J, Larsen RH. Advantage of lutetium-177 versus radioiodine immunoconjugate in targeted radionuclide therapy of B-cell tumors. Anticancer Res. 2014;34:3263-3269.

10. Tagawa ST, Milowsky MI, Morris M, et al. Phase II study of lutetium-177-labeled anti-prostate-specific membrane antigen monoclonal antibody J591 for metastatic castration-resistant prostate cancer. Clin Cancer Res. 2013;19:5182-5191.

11. Schoffelen R, Boerman OC, Goldenberg DM, et al. Development of an imagingguided CEA-pretargeted radionuclide treatment of advanced colorectal cancer: first clinical results. Br J Cancer. 2013;109:934-942.

12. Zaknun JJ, Bodei L, Mueller-Brand J, et al. The joint IAEA, EANM, and SNMMI practical guidance on peptide receptor radionuclide therapy (PRRNT) in neuroendocrine tumours. Eur J Nucl Med Mol Imaging. 2013;40:800-816.

13. Jødal L. Beta emitters and radiation protection. Acta Oncol. 2009;48:308-313.

14. Repetto-Llamazares AH, Larsen RH, Mollatt C, Lassmann M, Dahle J. Biodistribution and dosimetry of ${ }^{177} \mathrm{Lu}$-tetulomab, a new radioimmunoconjugate for treatment of non-Hodgkin lymphoma. Curr Radiopharm. 2013;6:20-27.

15. Blakkisrud J, Løndalen A, Dahle J, et al. Red marrow absorbed dose for nonHodgkin's lymphoma patients treated with the novel anti-CD37 antibody radionuclide conjugate ${ }^{177}$ Lu-lilotomab satetraxetan. J Nucl Med. 2017;58:55-61.

16. Blakkisrud J, Løndalen A, Martinsen ACT, et al. Tumor absorbed dose for nonHodgkin's lymphoma patients treated with the novel anti-CD37 antibody radionuclide conjugate ${ }^{177} \mathrm{Lu}$-lilotomab satetraxetan. J Nucl Med. 2017;58:48-54.

17. Dewaraja YK, Frey EC, Sgouros G, et al. MIRD pamphlet no. 23: quantitative SPECT for patient-specific 3-dimensional dosimetry in internal radionuclide therapy. J Nucl Med. 2012;53:1310-1325.

18. Marks LB, Yorke ED, Jackson A, et al. The use of normal tissue complication probability (NTCP) models in the clinic. Int J Radiat Oncol Biol Phys. 2010; 76:10-19. 
19. Sandström M, Garske-Roman U, Granberg D, et al. Individualized dosimetry of kidney and bone marrow in patients undergoing ${ }^{177} \mathrm{Lu}$-DOTA-octreotate treatment. J Nucl Med. 2013;54:33-41.

20. Kolstad A, Madsbu U, Beasley M, et al. Efficacy and safety results of Betalutin ${ }^{\circledR}$ (177-Lu-DOTA-HH1) in a phase $1 / 2$ study of patients with non-Hodgkin B-cell lymphoma (NHL). Presented at: AACR Annual Meeting; New Orleans, Louisiana; 2016.

21. Svensson J, Hagmarker L, Magnander T, Wangberg B, Bernhardt P. Radiation exposure of the spleen during ${ }^{177} \mathrm{Lu}$-DOTATATE treatment and its correlation with haematological toxicity and spleen volume. EJNMMI Phys. 2016;3:15.

22. Kulkarni HR, Prasad V, Schuchardt C, Baum RP. Is there a correlation between peptide receptor radionuclide therapy-associated hematological toxicity and spleen dose? Recent Results Cancer Res. 2013;194:561-566.

23. Fisher DR, Shen S, Meredith RF. MIRD dose estimate report no. 20: radiation absorbed-dose estimates for ${ }^{111} \mathrm{In}$ - and ${ }^{90}$ Y-ibritumomab tiuxetan. $\mathrm{J}$ Nucl Med. 2009;50:644-652.

24. Vose JM, Wahl RL, Saleh M, et al. Multicenter phase II study of iodine-131 tositumomab for chemotherapy-relapsed/refractory low-grade and transformed low-grade B-cell non-Hodgkin's lymphomas. J Clin Oncol. 2000;18: 1316-1323.

25. Forrer F, Oechslin-Oberholzer C, Campana B, et al. Radioimmunotherapy with ${ }^{177}$ Lu-DOTA-rituximab: final results of a phase I/II study in 31 patients with relapsing follicular, mantle cell, and other indolent B-cell lymphomas. $\mathrm{J} \mathrm{Nucl}$ Med. 2013;54:1045-1052.
26. Eary JF, Press OW, Badger CC, et al. Imaging and treatment of B-cell lymphoma. J Nucl Med. 1990;31:1257-1268.

27. Kaminski MS, Zasadny KR, Francis IR, et al. Iodine-131-anti-B1 radioimmunotherapy for B-cell lymphoma. J Clin Oncol. 1996;14:1974-1981.

28. Zevalin summary of product characteristics. European Medicines Agency website. http://www.ema.europa.eu/docs/en_GB/document_library/EPAR_-_Product_ Information/human/000547/WC500049469.pdf. Accessed April 20, 2017.

29. Wiseman GA, Kornmehl E, Leigh B, et al. Radiation dosimetry results and safety correlations from ${ }^{90}$ Y-ibritumomab tiuxetan radioimmunotherapy for relapsed or refractory non-Hodgkin's lymphoma: combined data from 4 clinical trials. J Nucl Med. 2003;44:465-474.

30. Assié K, Dieudonne A, Gardin I, Buvat I, Tilly H, Vera P. Comparison between 2D and $3 \mathrm{D}$ dosimetry protocols in ${ }^{90} \mathrm{Y}$-ibritumomab tiuxetan radioimmunotherapy of patients with non-Hodgkin's lymphoma. Cancer Biother Radiopharm. 2008;23:53-64.

31. Ljungberg M, Celler A, Konijnenberg MW, Eckerman KF, Dewaraja YK, Sjögreen-Gleisner K. MIRD pamphlet no. 26: joint EANM/MIRD guidelines for quantitative ${ }^{177} \mathrm{Lu}$ SPECT applied for dosimetry of radiopharmaceutical therapy. J Nucl Med. 2016;57:151-162.

32. Bexxar: highlights of prescribing information. GSK Source website. https:// www.gsksource.com/pharma/content/dam/GlaxoSmithKline/US/en/Prescribing_ Information/Bexxar/pdf/BEXXAR.PDF. Published 2013. Accessed April 20, 2017.

\section{Errata}

In the article "The SNMMI and EANM Practice Guideline for Small-Bowel and Colon Transit 1.0*" by Maurer et al. ( $\mathrm{N} \mathrm{Nucl} \mathrm{Med.} \mathrm{2013;54:2004-2013),} \mathrm{Table} 1$ (column 2) incorrectly states that the administered activity for ${ }^{99 \mathrm{~m}} \mathrm{Tc}$-labeled nonabsorbable markers (solids) is $18.5-37 \mathrm{MBq} / \mathrm{kg}(0.5-1.0 \mathrm{mCi} / \mathrm{kg})$ and the administered activity for ${ }^{111}$ In-labeled liquids (DTPA) is $3.7-37 \mathrm{MBq} / \mathrm{kg}(0.1-1.0 \mathrm{mCi} / \mathrm{kg})$. The correct activities are $18.5-37 \mathrm{MBq}(0.5-1.0 \mathrm{mCi})$ and 3.7-37 MBq $(0.1-1.0 \mathrm{mCi}$ ), respectively. In addition, Figure 1 (options $\mathrm{A}, \mathrm{B}$, and $\mathrm{C}$ ) incorrectly states that the range for $300 \mathrm{~mL}{ }^{111} \mathrm{In}$ water is $3.7-7.4 \mathrm{MBq}$. The correct range is $3.7-37 \mathrm{MBq}$. We regret the errors.

In the article "Evaluation of Radiopharmaceutical Adverse Reaction Reports to the British Nuclear Medicine Society from 2007 to 2016" by Kennedy-Dixon et al. (J Nucl Med. 2017;58:2012-2014), the Discussion section incorrectly says that "Silberstein (8) found 15 reports of flushing of the face and trunk within minutes of ${ }^{18} \mathrm{~F}-\mathrm{FDG}$ administration from 15 nuclear medicine facilities in the United States between 2007 and 2011." In fact, the cited Silberstein article ( $\mathrm{J} \mathrm{Nucl} \mathrm{Med.} \mathrm{2014;55:1308-1310)} \mathrm{stated} \mathrm{that} \mathrm{only} \mathrm{one} \mathrm{episode} \mathrm{of} \mathrm{facial} \mathrm{and} \mathrm{truncal} \mathrm{flushing} \mathrm{had}$ been reported during that interval. We regret the error. 